\title{
INDICADORES
}

\section{RANKINGS ISI DE LAS UNIVERSIDADES ESPAÑOLAS SEGÚN CAMPOS CIENTÍFICOS: DESCRIPCIÓN Y RESULTADOS}

\author{
Daniel Torres-Salinas, Emilio Delgado-López-Cózar, José García-Moreno- \\ Torres y Francisco Herrera
}

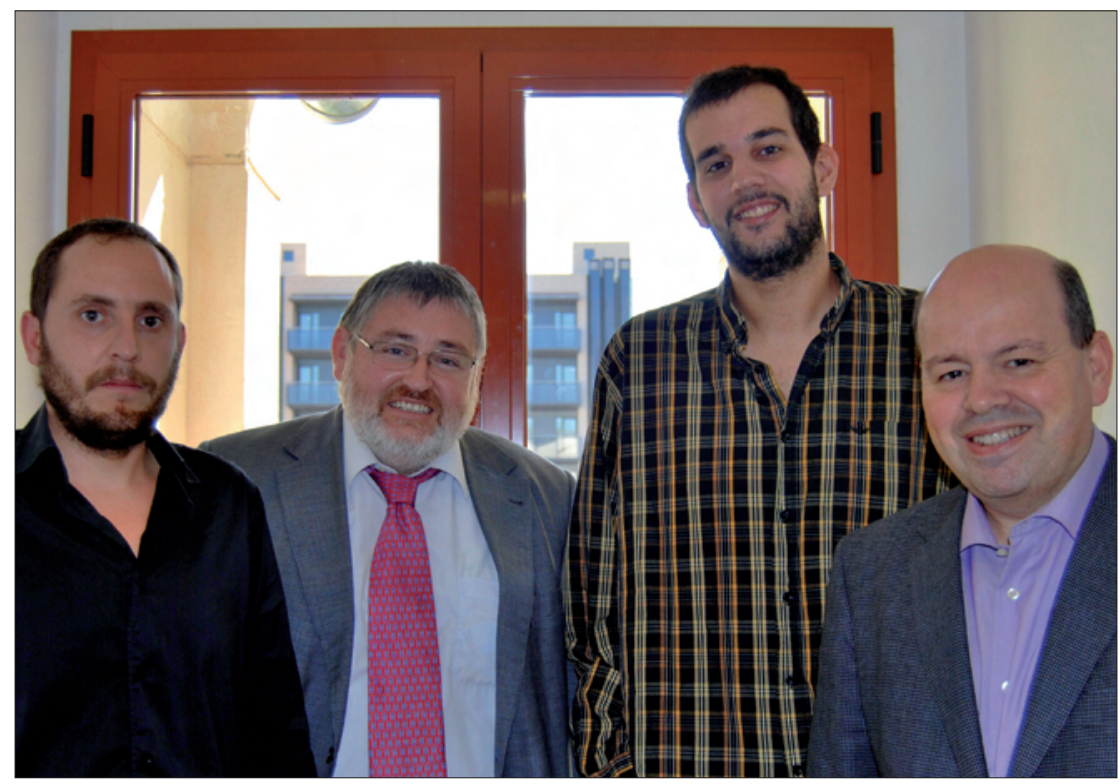

Daniel Torres-Salinas es doctor en documentación científica y trabaja como técnico de gestión de la investigación en el Centro de Investigación Médica Aplicada de la Universidad de Navarra, donde realiza auditorías sobre la calidad y el impacto de la investigación. Es miembro del Grupo EC3 (Evaluación de la Ciencia y de la Comunicación Científica) de la Universidad de Granada donde participa en diferentes proyectos. Es miembro del think tank ThinkEPI.
Grupo EC3 (Evaluación de la Ciencia y la Comunicación Científica) Universidad de Granada
Centro de Investigación Médica Aplicada Universidad de Navarra 31008 Pamplona, España torressalinas@gmail.com

Emilio Delgado-López-Cózar es catedrático de metodología de la investigación en la Facultad de Comunicación y Documentación de la Universidad de Granada y miembro del Grupo EC3. Sus líneas de investigación se centran en la evaluación de las revistas científicas, el estudio de la investigación en ByD, y la evaluación del rendimiento investigador. Es promotor de sistemas y herramientas para la evaluación científica como In-Recs/In-Recj, Científica, Rankings ISI de Universidades.

\author{
Grupo EC3 (Evaluación de la Ciencia y la Comunicación Científica) \\ Facultad de Comunicación y Documentación \\ Universidad de Granada \\ Campus de la Cartuja, s/n. 18071 Granada, España \\ edelgado@ugr.es
}

José García-Moreno-Torres es estudiante de doctorado en el Departamento de Ciencias de la Computación e Inteligencia Artificial de la Universidad de Granada, dentro del Grupo SCI2S (Soft Computing and Intelligent Information Syste$m s)$. En la actualidad sus intereses de investigación incluyen fractura de datos, clasificación con conjuntos de datos no balanceados, aprendizaje multi-instancia y bibliometría.

Depto. de Ciencias de la Computación e Inteligencia Artificial Universidad de Granada 18071 Granada, España jose.garcia.mt@decsai.ugr.es

Francisco Herrera es catedrático del Departamento de Ciencias de la Computación e Inteligencia Artificial de la Universidad de Granada y director del Grupo de investigación Soft Computing y Sistemas de Información Inteligentes. Ha dirigido 22 tesis doctorales y publicado más de 180 artículos en revistas internacionales en las áreas de inteligencia computacional, minería de datos y bibliometría. Ha dirigido varios proyectos centrados en el desarrollo de técnicas de inteligencia computacional y sus aplicaciones.

Departamento de Ciencias de la Computación e Inteligencia Artificial ETS de Ingenierías Informática y de Telecomunicación Universidad de Granada. 18071 Granada herrera@decsai.ugr.es 


\title{
Resumen
}

Se describen los "Rankings ISI de las universidades españolas según campos del conocimiento" y se presentan algunos de sus resultados. Una de las características esenciales de estos nuevos rankings, basados en los productos de ThomsonReuters, es la organización en 12 campos del conocimiento diferentes. Como criterio de ordenación de las universidades se ha empleado el IFQ2A-Index que sintetiza información de 6 indicadores bibliométricos distintos. En los resultados generales derivados del ranking se presentan indicadores globales del sistema universitario español que muestran un crecimiento sostenido de su producción científica pero un estancamiento en el incremento de la visibilidad de las revistas. Asimismo el análisis específico de los resultados del ranking manifiesta el dominio de las universidades catalanas en los doce campos del conocimiento analizados durante el quinquenio 2005-2009.

\section{Palabras clave}

Rankings, Indicadores bibliométricos, IFQ2A-Index, Producción científica, Análisis de citas, Universidades, Dominios temáticos, España, Thomson-Reuters.

\section{Title: ISI rankings of universities in Spain by scientific field}

\begin{abstract}
The "ISI rankings of the spanish universities by scientific fields" report is described, and some of the results are presented. One of the essential characteristics of this new ranking, based on Thomson-Reuters products, is its organization into 12 fields of knowledge. The IFQ2A-Index indicator was used to sort the universities, synthesizing information from 6 bibliometric indicators. The results section presents some total indicators for the Spanish university system, showing a steady growth of scientific production but almost no change in the visibility of the journals. Furthermore, a detailed analysis of the results shows the dominance of Catalan universities in the 12 fields of knowledge analyzed during the period 2005-2009.
\end{abstract}

\section{Keywords}

Rankings, Bibliometric indicators, IFQ2A-Index, Scientific output, Citation analysis, Universities, Subject domains, Spain, Thomson-Reuters.

Torres-Salinas, Daniel; Delgado-López-Cózar, Emilio; García-Moreno-Torres, José; Herrera, Francisco. "Rankings ISI de las universidades españolas según campos científicos: descripción y resultados". El profesional de la información, 2010, enero-febrero, v. 20, n. 1, pp. 111-118.

DOI: 10.3145/epi.2011.ene.14

\section{Introducción}

Desde el lanzamiento del Academic Ranking of World Universities $(A R W U)^{1}$ en 2003 los rankings de universidades han proliferado considerablemente. Junto al ARWU (o Ranking Shangai, como también se le conoce) han surgido otras propuestas que han logrado cierto grado de aceptación como es el caso del Leiden Ranking ${ }^{2}$, QS World University Ranking ${ }^{3}$, Times Higher Education Ranking ${ }^{4}, \mathrm{CHE}$-Ranking $^{5}$, Scimago Institutions Rankings ${ }^{6}$ y el Ranking Web of World Universities ${ }^{7}$. Algunos de ellos son en la actualidad referencia indiscutible para conocer el rendimiento científico y académico de las instituciones de educación superior y fuente de información esencial en numerosas universidades y países para la toma de decisiones en el campo de la política científica (Marginson; Van-der-Wender, 2007; Aguillo et al., 2010). Sin embargo desde sus inicios estos rankings han sido criticados en la bibliografía científica por una serie de cuestiones entre las que destacan los criterios de selección de indicadores, la ponderación y peso que se otorga a los mismos, los errores en la toma y procesamiento de los datos o la falta de transparencia y replicabilidad de sus resultados (Bordons et al., 2010; Liu; Cheng, 2005; Van-Raan, 2005).
Un problema añadido en el caso de España es que no reflejan adecuadamente nuestro sistema universitario al ofrecer una imagen fragmentada del mismo. Al ser clasificaciones con un carácter internacional se limitan al top mundial por lo que la mayor parte de nuestras instituciones no alcanzan los mínimos para figurar en ellos. Así, por ejemplo, en la última edición de $A R W U$ y de $Q S$ tan sólo identificamos 10 universidades españolas en el top500 mundial. Por esta razón es discutible hasta qué punto consiguen reflejar el estado de las universidades españolas. Para resolver este problema la solución más idónea es plantear un ranking dedicado exclusivamente a universidades españolas, como los presentados en la Calidad de las universidades en España (Buesa; Heijs; Kahwash, 2009) en el Ranking global de productividad en investigación" (BuelaCasal et al., 2009) o en el Ranking general y por áreas de las instituciones universitarias españolas (Corera et al., 2010).

Sin embargo estos productos, que son relativamente recientes, también presentan problemas, entre los que se pueden apuntar los siguientes:

1) falta de actualización y continuidad en el tiempo (Buesa; Heijs; Kahwash, 2009);

2) exclusión de los centros privados (Buela-Casal et al., 2009); 
3) no tomar en consideración distintos campos científicos y especialización temática de las universidades (Buesa; Heijs; Kahwash, 2009; Buela-Casal et al., 2009) o cuando se hace, tener sólo en cuenta determinadas áreas como la medicina, la química o la física y astronomía (Corera et al., 2010);

4) emplear indicadores bibliométricos muy primarios (publicaciones y citas) sin considerar otras variables que pueden cualificar los resultados (Buesa; Heijs; Kahwash, 2009; Buela-Casal et al., 2009);

5) seleccionar series temporales demasiado cortas, como anualidades (Buesa; Heijs; Kahwash, 2009; Buela-Casal et al., 2009) o emplear series amplias pero poco actualizadas (Corera et al., 2010);

6) elección de fuentes de información cuyos criterios de inclusión de las revistas es dudosa como Scopus (Corera et al., 2010).

Por ello se hace necesaria la generación de nuevas propuestas que superen algunos de los problemas señalados y permitan mapear las universidades españolas. Con este propósito se han publicado los Rankings ISI de las universidades españolas según campos del conocimiento, con un método de ordenación que sintetiza seis indicadores bibliométricos. Este producto intenta cubrir varias de las limitaciones citadas, ya que abarca tanto centros públicos como privados, se divide en 12 campos del conocimiento científico para captar la especialización de los centros y, en último lugar, utiliza períodos cronológicos amplios para garantizar la consistencia de los indicadores. Es por tanto un ranking de investigación, que emplea los productos de Thomson-Reuters (Web of Science-WoS y Journal Citation Reports-JCR), referentes indiscutibles de la política científica nacional (Ruiz-Pérez; Delgado-López-Cózar; Jiménez-Contreras, 2010).

El doble objetivo que se plantea en este trabajo es, en primer lugar, realizar un repaso de las principales características y funciones de los Rankings ISI..., que fueron publicados en octubre de 2010. En segundo lugar se mostrarán resultados originales derivados de sus datos divididos en dos partes: por un lado los principales indicadores del sistema universitario español en su conjunto y por otro lado un análisis de los rankings del quinquenio 2005-2009. Por tanto este artículo se puede considerar un complemento de los diferentes materiales disponibles en el site de Rankings $I S I^{6}$.

\section{Configuración de los campos y diseño del IFQ ${ }^{2} A-I n d e x$}

Una de las características esenciales de Rankings ISI es su enfoque y presentación mediante 12 campos de conocimiento y no por grandes ramas de conocimiento que diluyen los distintos perfiles de investigación que exhiben las universidades, de manera que pueda captarse mejor en qué temáticas son más activas e influyentes. Dichos campos se han planteado a partir de la agregación y asignación de las 225 categorías temáticas del JCR a cada uno de los 12 campos, por tanto, se han configurado a partir de la categoría donde se indexan las revistas. De esta forma, por ejemplo, las revistas pertenecientes a la categoría Medicine general \& internal han sido asignadas al campo Medicina clínica, farmacia y farmacología. Es pues una clasificación basada en la especialización de las revistas que sigue una perspectiva similar a la empleada en otros rankings.

Como medida final de ordenación de las universidades en los distintos campos se diseñó un nuevo indicador denominado IFQ ${ }^{2} A$-Index (Institutional field quantitative-qualitative index analysis index). Se define formalmente como un indicador bidimensional que compara y ordena un conjunto de universidades en función de su producción e impacto científico en un campo. Tiene un carácter bidimensional ya que es resultado de la combinación de dos indicadores compuestos que resumen las dimensiones cuantitativas y cualitativas de las publicaciones científicas indexadas en las bases de datos de Thomson-Reuters.

Para la construcción de dichas dimensiones se seleccionó un total de seis indicadores bibliométricos consolidados:

- Número de trabajos citables indexados en el JCR (ndoc);

- Número de citas (ncit);

- Índice $h$ (h-index);

- Porcentaje de documentos en el primer cuartil (\%1Q);

- Promedio de citas (pcit) y

- Porcentaje de altamente citados (topcit).

Una vez seleccionados estos indicadores fueron agrupados en función de su correlación en dos conjuntos diferentes. El primero se corresponde con una dimensión cuantitativa (ndoc, ncit, h-index) compuesta por medidas muy relacionadas con el tamaño de las instituciones; el segundo responde a una dimensión cualitativa (\%1Q, pctic, topcit) y lo conforman indicadores independientes del tamaño de las universidades.

Para el cálculo final del IFQ ${ }^{2} A$-Index los indicadores para cada universidad se normalizan entre 0-1 tomando como referencia la universidad que alcanza el valor más alto. A continuación se calculan las dos dimensiones establecidas a partir de la media geométrica de los valores normalizados de los indicadores que las componen. En un último paso el múltiplo final de los valores de la dimensión cuantitativa y cualitativa nos da como resultado el IFQ ${ }^{2} A$-Index. El objetivo de este indicador es evitar por un lado los rankings unidimensionales como los basados en producción y por otro buscar un equilibro entre la cantidad y la calidad de las publicaciones de las universidades, evitando así que las universidades con un menor número de trabajos JCR pero de gran calidad queden relegadas a bajas posiciones.

\section{Descripción y contenidos del website Rankings ISI}

Los Rankings ISI de las universidades españolas según campo científico pueden ser consultados online ${ }^{8}$. En su web se ofrecen para cada uno de los 12 campos científicos analizados dos rankings en formato pdf que responden a dos períodos cronológicos: 2000-2009 y 2005-2009. Cada uno de estos archivos pdf consta de dos páginas; en la primera se presentan los valores de las dimensiones cuantitativas y cualitativas que obtiene cada universidad y el valor final del $I F Q^{2} A-$ Index, y en la segunda se muestran como apoyo los valores brutos y normalizados de los seis indicadores seleccionados. De esta forma no sólo se facilita información de interés para 
las universidades sino que además permite que los diferentes cálculos y procesos llevados a cabo puedan ser reproducidos. La información de los rankings se complementa con unos gráficos interactivos que representan las dos dimensiones analizadas. Asimismo, junto a los archivos pdf todos los datos generados y los rankings para los 12 campos y los 2 períodos pueden ser descargados conjuntamente en un archivo excel. Junto a estos materiales se puede encontrar un resumen de posiciones en el que se presenta una matriz universidad-campo con el rango ocupado por cada centro. Por último también está disponible un documento donde se explica extensamente toda la metodología, como el cálculo matemático del IFQ ${ }^{2} A$-Index o el detalle de las categorías $J C R$ que conforman cada uno de los campos científicos.

http://rankinguniversidades.es

\section{Top3}

Es el número o porcentaje de documentos citables publicados en las revistas que ocupan alguna de las tres primeras posiciones de cualquiera de las categorías temáticas de los Journal Citation Reports. Es como el primer cuartil pero sólo tomando las tres primeras revistas.

\section{Resultados}

\subsection{La universidad española en su conjunto}

En el gráfico 1 se muestra la producción en revistas JCR de las universidades. Se han publicado 211.038 trabajos citables $J C R$ en la última década con un crecimiento sostenido. Entre el año 2000 y el 2009 la producción científica ha aumentado un $90 \%$ pasándose de los 15.442 papers del 2000 a los 29.429 de 2009. En relación con el impacto, el número de documentos brutos indexados en revistas del primer cuartil y top3 también crece entre 2000 y 2009 un $125 \%$ y $97 \%$ respectivamente. Sin embargo, el porcentaje de documentos en el primer cuartil sólo se ha incrementado del $45 \%$ de comienzos de década al 53\% de 2009. Si hablamos de porcentaje de revistas top3, es decir la élite de las revistas científicas, sistemáticamente todos los años el porcentaje se mantiene en el $10 \%$. Estos resultados manifiestan que la universidad española ha conseguido aumentar de manera sobresaliente la cantidad de trabajos en publicaciones de alcance internacional pero revelan una mayor dificultad a la hora de incrementar su presencia en las revistas de mayor excelencia e impacto.

Si atendemos a la especialización del sistema universitario en el quinquenio 2005-2009 (gráfico 2) a través de los 12 campos científicos definidos en los Rankings ISI, Medicina clínica, farmacia y farmacología acapara el $21 \%$ de la producción científica. Le siguen la Química, la Ingeniería y las Ciencias biológicas todas con el $18 \%$. Sólo estos 4 campos ya representan el $75 \%$ de la producción de las universidades. Las que menos documentos aportan son las disciplinas cercanas a las ciencias sociales (Psicología, Economía y Otras ciencias sociales) que conjuntamente suman el $8 \%$ del total.

En relación con el impacto, cuatro campos consiguen publicar entre el 50\%-60\% de sus documentos en el primer cuartil (Química, Ingeniería, Física y Ciencias agrarias), volviendo a ser las ciencias sociales las que menos impacto alcanzan indexando el $20 \%-30 \%$ de sus documentos en el primer cuartil.

En la tabla 1 se presenta el Índice de actividad temática (ver definición a pie de tabla) de las 28 principales universidades del período 2005-2009, entendiendo como tales aquellas que se han situado entre la posición 1-10 en alguno de los 12 campos analizados. En primer lugar habría que reseñar que en todos los campos encontramos entre 9 y 14 universidades con una especialización mayor que el conjunto de la universidad española, es decir con valores superiores a 1. El único campo que no sigue este patrón es Medicina clínica, farmacia y farmacología del que sólo encontramos especialización en 5 universidades. Por otro lado los índices de especialización más altos los localizamos en Ciencias agrarias en la Universitat de Lleida $(5,10)$, en Economía en la Universidad Carlos III de Madrid $(7,26)$, en Medicina clínica en la Universidad de Navarra $(2,13)$ y en Ingeniería y TIC

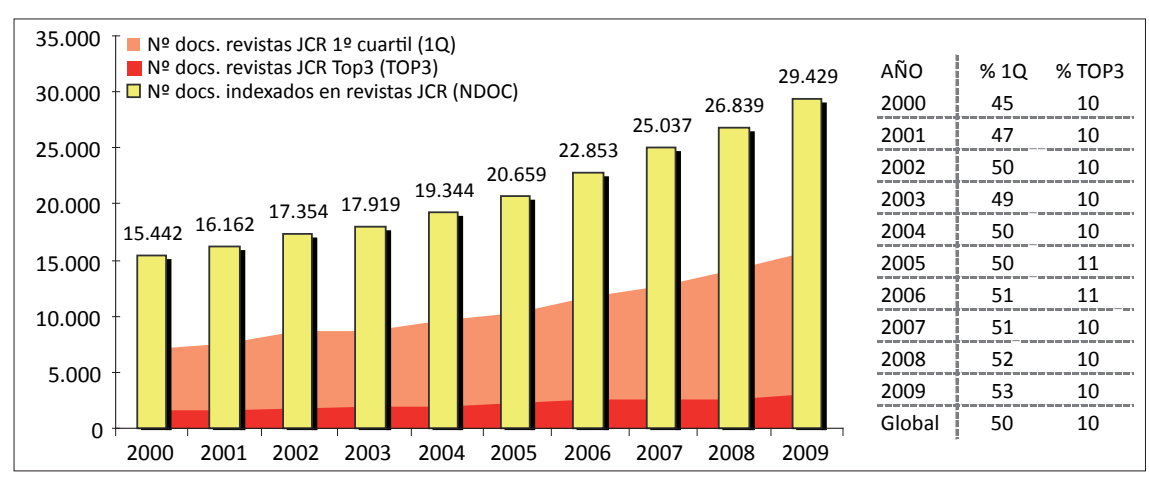

Gráfico 1. Evolución de la producción e impacto del sistema universitario español en revistas indexadas en los JCR. Período 2000-2009

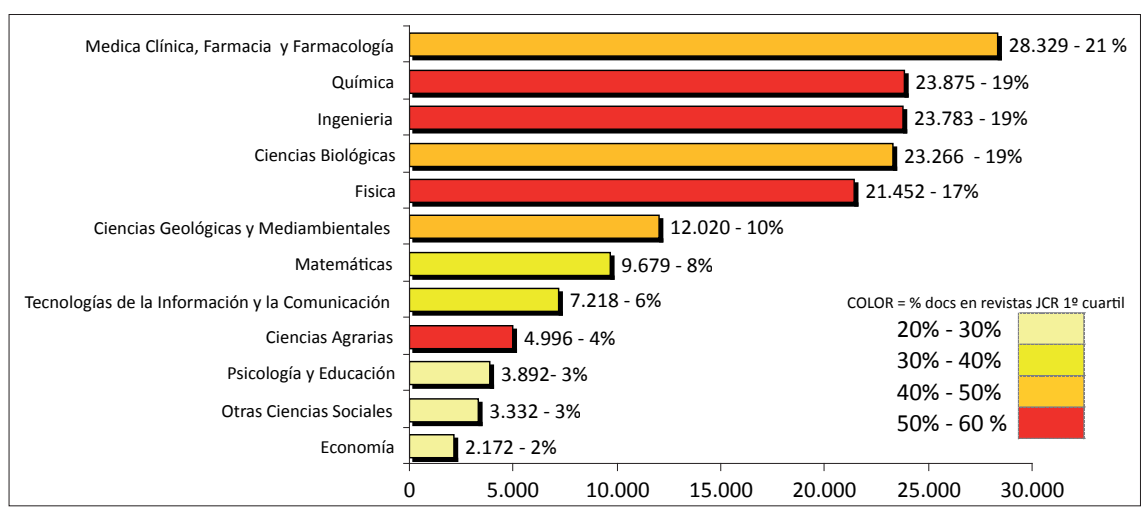

Gráfico 2. Producción e impacto del sistema universitario español en revistas indexadas en los JCR por campo científico. Período 2005-2009 


\begin{tabular}{|c|c|c|c|c|c|c|c|c|c|c|c|c|}
\hline UNIVERSIDAD & MAT & FIS & QUIM & $\mathrm{BIO}$ & MED & GEO & AGR & ING & TIC & PSI & ECO & OCS \\
\hline Alicante & 0,75 & 1,09 & 1,51 & 0,56 & 0,58 & 1,27 & 0,50 & 1,03 & 0,93 & 0,48 & 2,29 & 1,19 \\
\hline Autònoma de Barcelona & 0,75 & 1,03 & 0,68 & 1,13 & 1,08 & 0,94 & 1,04 & 0,63 & 0,37 & 0,82 & 1,21 & 1,04 \\
\hline Autónoma de Madrid & 0,79 & 1,55 & 0,74 & 1,26 & 0,88 & 0,47 & 0,25 & 0,52 & 0,43 & 1,26 & 0,44 & 0,52 \\
\hline Barcelona & 0,44 & 0,93 & 0,96 & 1,13 & 1,24 & 1,00 & 0,42 & 0,45 & 0,23 & 1,06 & 0,77 & 0,84 \\
\hline Cantabria & 1,17 & 1,71 & 0,24 & 0,53 & 0,85 & 0,86 & 0,15 & 1,23 & 1,15 & 0,33 & 1,08 & 0,96 \\
\hline Carlos III de Madrid & 3,01 & 1,49 & 0,20 & 0,07 & 0,09 & 0,24 & 0,04 & 1,76 & 3,33 & 0,35 & 7,26 & 3,45 \\
\hline Castilla la Mancha & 1,08 & 0,63 & 1,03 & 1,09 & 0,54 & 1,48 & 1,56 & 1,39 & 1,71 & 0,77 & 0,71 & 0,63 \\
\hline Complutense de Madrid & 0,90 & 1,14 & 0,82 & 1,05 & 0,87 & 1,08 & 0,73 & 0,75 & 0,59 & 1,04 & 0,57 & 1,02 \\
\hline Córdoba & 0,28 & 0,30 & 1,40 & 1,84 & 0,76 & 0,90 & 4,29 & 0,96 & 0,75 & 0,43 & 0,05 & 0,18 \\
\hline Granada & 1,60 & 0,76 & 0,73 & 0,81 & 0,73 & 1,67 & 0,68 & 0,70 & 1,30 & 2,12 & 0,46 & 1,49 \\
\hline Islas Baleares & 0,54 & 1,46 & 0,78 & 1,21 & 0,53 & 1,95 & 0,35 & 0,54 & 0,86 & 1,07 & 1,10 & 1,12 \\
\hline La Laguna & 1,42 & 1,08 & 1,12 & 0,99 & 0,70 & 1,11 & 0,69 & 0,67 & 0,65 & 2,46 & 0,49 & 0,77 \\
\hline Lleida & 0,97 & 0,28 & 0,67 & 1,47 & 0,60 & 1,21 & 5,10 & 1,28 & 0,70 & 1,20 & 0,44 & 0,52 \\
\hline Murcia & 1,25 & 0,40 & 0,84 & 1,70 & 0,88 & 0,69 & 1,75 & 0,64 & 0,75 & 1,74 & 0,98 & 1,18 \\
\hline Navarra & 0,42 & 0,26 & 0,37 & 1,00 & 2,13 & 0,27 & 0,49 & 0,70 & 0,44 & 0,44 & 2,02 & 1,49 \\
\hline Oviedo & 0,78 & 0,73 & 1,33 & 0,86 & 0,66 & 1,03 & 0,60 & 1,10 & 0,99 & 1,82 & 1,03 & 0,93 \\
\hline País Vasco & 0,75 & 1,51 & 1,37 & 0,75 & 0,51 & 0,79 & 0,38 & 1,14 & 0,76 & 1,09 & 0,83 & 1,09 \\
\hline Politècnica de Catalunya & 2,17 & 1,46 & 0,63 & 0,28 & 0,19 & 1,42 & 0,37 & 2,09 & 2,91 & 0,22 & 0,18 & 0,30 \\
\hline Politécnica de Madrid & 1,15 & 1,62 & 0,31 & 0,47 & 0,19 & 1,00 & 3,08 & 2,04 & 2,35 & 0,46 & 0,37 & 0,52 \\
\hline Politécnica de Valencia & 1,67 & 0,97 & 1,17 & 0,72 & 0,19 & 0,73 & 1,47 & 2,04 & 2,28 & 0,45 & 0,20 & 0,76 \\
\hline Pompeu Fabra & 1,19 & 0,23 & 0,20 & 1,51 & 1,31 & 0,30 & 0,00 & 0,20 & 1,68 & 0,89 & 6,22 & 3,41 \\
\hline Rovira i Virgili & 0,63 & 0,70 & 1,78 & 0,87 & 0,73 & 0,94 & 0,42 & 1,25 & 1,19 & 0,96 & 1,01 & 1,33 \\
\hline Salamanca & 0,82 & 0,95 & 0,75 & 1,31 & 1,08 & 0,86 & 0,35 & 0,58 & 0,45 & 1,33 & 0,99 & 1,22 \\
\hline Santiago de Compostela & 0,83 & 0,92 & 1,38 & 1,08 & 0,89 & 0,85 & 1,75 & 0,73 & 0,42 & 0,83 & 0,42 & 0,40 \\
\hline Sevilla & 1,80 & 0,98 & 0,91 & 0,93 & 0,52 & 0,79 & 0,91 & 1,29 & 1,33 & 0,87 & 0,52 & 0,74 \\
\hline Valencia & 0,72 & 1,38 & 1,20 & 0,90 & 0,86 & 0,63 & 0,34 & 0,61 & 0,65 & 1,40 & 1,01 & 0,92 \\
\hline Vigo & 1,00 & 0,77 & 1,54 & 1,14 & 0,44 & 1,99 & 1,44 & 1,31 & 1,17 & 0,53 & 1,11 & 0,65 \\
\hline Zaragoza & 1,29 & 1,30 & 1,20 & 0,82 & 0,53 & 0,92 & 0,83 & 1,11 & 0,84 & 0,37 & 1,17 & 1,04 \\
\hline \multicolumn{13}{|c|}{$\begin{array}{l}\text { - Para los acrónimos de los campos científicos ver la nota } 9 \\
\text { - Comentarios: Sólo aparecen las universidades que han logrado situarse entre las posiciones 1-10 en alguno de los } 12 \text { campos analizados } \\
\text { - Cálculo del Índice de actividad temática (IAT). En primer lugar se calcula el porcentaje de documentos que las universidades publican en cada uno de los campos cien- } \\
\text { tíficos. A continuación dicho porcentaje se divide entre el porcentaje nacional. De esta forma un IAT inferior a } 1 \text { indica que la universidad presenta menor especializa- } \\
\text { ción que el sistema universitario nacional en su conjunto, un índice superior a } 1 \text { índica una mayor especialización. }\end{array}$} \\
\hline
\end{tabular}

Tabla 1. Índice de actividad temática de las principales universidades españolas para los 12 campos del conocimiento analizados en los Rankings ISI durante el período 2005-2009

en las tres grandes politécnicas (Madrid, Catalunya y Valencia). Este indicador confirma la idoneidad de un ranking por campos ya que el perfil de las universidades españolas es variado.

\subsection{Resumen de los resultados de los Rankings ISI en 2005-2009}

En la tabla 2 se presentan los resultados del ranking para el último quinquenio, mostrándose las tres primeras universidades en cada uno de los campos. Hay que señalar en primer lugar que son las universidades de Catalunya las que consiguen alcanzar la primera posición en todos los rankings. Así la Universitat Politècnica de Catalunya domina 2 campos científicos (Matemáticas, y Tecnología de la información y las comunicaciones), lo mismo que la Universitat Pompeu Fabra (Economía y Otras ciencias sociales). Igualmente la Universitat Autònoma de Barcelona consigue el primer puesto en Ciencias agrarias. El resto de primeras posiciones, un total de 7 campos, queda en manos de la Universitat de Barcelona. Este dominio de los centros catalanes es tan abrumador que incluso hay campos donde podemos localizar dos universidades catalanas entre las tres primeras posiciones como es el caso de Física, Ciencias biológicas, Medicina clínica farmacia y farmacología, Ciencias geológicas, Ingeniería, Economía y Otras ciencias sociales.
El otro gran eje científico de España, aunque muy alejado del anterior, es el de las universidades madrileñas que alcanzan sus mejores resultados con las segundas posiciones de la Universidad Autónoma de Madrid en Ciencias biológicas, de la Universidad Complutense en Ciencias agrarias y de la Universidad Carlos III en Otras ciencias sociales. Finalmente y dejando al margen los núcleos madrileños y catalanes sólo tres universidades más consiguen situarse en alguna de las tres primeras posiciones, destacando entre ellas la Universitat de València; esta institución despunta sobre todo en los campos científicos de Física y Química con sendas segundas posiciones, al igual que ocurre con la Universidad de Granada en Psicología y ciencias de la educación, y Tecnología de la información y las comunicaciones. Por último habría que mencionar entre las posiciones top el segundo puesto de la Universidade de Santiago de Compostela en Matemáticas.

En la tabla 3 se presenta un resumen de las posiciones que han logrado ocupar las universidades durante el quinquenio 2005-2009. Tan sólo 10 universidades de las 74 del sistema universitario consiguen aglutinar todas las posiciones que van del primer al cuarto puesto. Estas universidades, que coinciden en su mayoría con las ya reseñadas en la tabla 2, se configuran como el núcleo que consigue publicar en las bases de Thomson con mayor volumen e impacto; los resultados evidencian cómo las mejores universidades consiguen 


\begin{tabular}{|c|c|c|c|c|c|}
\hline \multicolumn{2}{|c|}{ Matemáticas } & \multicolumn{2}{|l|}{ Física } & \multicolumn{2}{|c|}{ Química } \\
\hline Politècnica de Catalunya & 0,544 & Barcelona & 0,806 & Barcelona & 0,740 \\
\hline Santiago de Compostela & 0,446 & Valencia & 0,784 & Valencia & 0,635 \\
\hline Politécnica de Valencia & 0,433 & Autònoma de Barcelona & 0,716 & Politécnica de Valencia & 0,495 \\
\hline \multicolumn{2}{|c|}{ Ciencias biológicas } & \multicolumn{2}{|c|}{ Medicina clínica, farmacia y farmacología } & \multicolumn{2}{|c|}{ Ciencias geológicas } \\
\hline Barcelona & 0,558 & Barcelona & 0,806 & Barcelona & 0,830 \\
\hline Autónoma de Madrid & 0,485 & Autònoma de Barcelona & 0,393 & Autònoma de Barcelona & 0,630 \\
\hline Pompeu Fabra & 0,358 & Autónoma de Madrid & 0,361 & Granada & 0,455 \\
\hline \multicolumn{2}{|c|}{ Ciencias agrarias } & \multicolumn{2}{|c|}{ Ingeniería } & \multicolumn{2}{|c|}{ Tecnología información y comunicaciones } \\
\hline Autònoma de Barcelona & 0,504 & Barcelona & 0,764 & Politècnica de Catalunya & 0,607 \\
\hline Complutense de Madrid & 0,367 & Autònoma de Barcelona & 0,704 & Granada & 0,385 \\
\hline Santiago de Compostela & 0,358 & Complutense de Madrid & 0,650 & Valencia & 0,382 \\
\hline \multicolumn{2}{|c|}{ Psicología y ciencias de la educación } & \multicolumn{2}{|c|}{ Economía } & \multicolumn{2}{|c|}{ Otras ciencias sociales } \\
\hline Barcelona & 0,900 & Pompeu Fabra & 0,835 & Barcelona & 0,541 \\
\hline Granada & 0,482 & Autònoma de Barcelona & 0,485 & Carlos III de Madrid & 0,463 \\
\hline Autónoma de Madrid & 0,450 & Carlos III de Madrid & 0,431 & Autònoma de Barcelona & 0,424 \\
\hline
\end{tabular}

Tabla 2. Universidades que han ocupado las tres primeras posiciones en función de su IFQ²A-Index según campo científico. Período $2005-2009$

grados de excelencia en casi todos los campos que publican mientras que el resto de centros apenas si consiguen destacarse a pesar de la desagregación por campos científicos. Esta situación es un síntoma de la clara polarización del sistema ya que el resto de universidades españolas sólo entra en juego si empezamos a considerar las quintas posiciones, donde empiezan a destacarse universidades como Sevilla, Vigo, Zaragoza, Murcia, Salamanca o Navarra, esta última único centro privado que consigue alguna posición relevante. A este segundo núcleo le seguirían otras ocho universidades que al menos han conseguido alcanzar una posición entre las diez primeras, como Cantabria, Castilla la Mancha, La Laguna o Lleida.

\section{Consideraciones finales}

En este trabajo se han sintetizado los principales resultados de los Rankings ISI. Es necesario en primer lugar subrayar, para su correcta interpretación, que este ranking se limita al ámbito de la investigación y concretamente a las variables que miden la capacidad para generar conocimiento y su impacto científico. No se emplean por tanto otras variables ligadas a la investigación (innovación o transferencia) y, menos aún, a dimensiones tan importantes como la docencia. Por tanto, las inferencias y deducciones deben limitarse a la producción científica y de ésta a la que tiene un carácter más internacional. Asimismo, al utilizar las bases de datos Web of Science como fuente de información se heredan también algunos de sus problemas como la menor cobertura de áreas como humanidades y ciencias sociales (Moed, 2005), factor a tener en cuenta para entender el porqué algunas universidades pueden no estar lo suficientemente representadas.

Por otro lado esta primera edición se ha organizado en campos lo suficientemente específicos para que puedan ser de utilidad a la hora de determinar las áreas de especialización de las universidades. Sin embargo mantienen todavía un carácter genérico ya que en la mayor parte de los casos los campos se han configurado como una agregación de espe- cialidades. Muchas de ellas tienen comportamientos bibliométricos (ciclos de publicación, ritmos de envejecimiento) y comunidades científicas lo suficientemente compactas y características para que puedan ser consideradas de forma separada. Por ello uno de los objetivos de próximas ediciones es la publicación no sólo de campos sino también de especialidades. En este contexto se prevé la creación de un ranking específico con una metodología ad hoc que contemple el campo de las humanidades.

Respecto a los resultados obtenidos a nivel nacional, se confirma el buen nivel de publicación de la universidad española con un porcentaje de trabajos en el primer cuartil que supera en 2009 el 50\%. Los datos están en consonancia por los obtenidos por Delgado-López-Cózar, Jiménez-Contreras y Ruiz-Pérez (2009) para 2003-2007 tanto en lo relativo al crecimiento de la producción como al impacto. Sin embargo se revela una mayor dificultad para crecer en publicaciones en las revistas que conforma la élite, el porcentaje en revistas top3 está estancado en el 10\%, y esta puede ser la razón de que nuestras tasas de citación no sean equiparables a las potencias científicas que podemos considerar excelentes (Suiza, EUA, Holanda, etc.) (Delgado-López-Cózar; JiménezContreras; Ruiz-Pérez, 2009). Podemos hablar, por tanto, de un modelo de crecimiento científico basado más en la cantidad que en la calidad.

En relación con los rankings, el núcleo de universidades que consiguen significarse por conseguir posiciones relevantes en diversos campos durante el quinquenio 2005-2009 (Barcelona, Politècnica de Catalunya, Autònoma de Barcelona, Politécnica de Madrid, Valencia, Pompeu Fabra, Complutense, Granada, Santiago de Compostela) son también aquellas que suelen figurar en rankings como $A R W U, C H E$ o THE, referencias indiscutibles. De esta forma los Rankings ISI son un complemento para las clasificaciones internacionales ya que apuntan hacia el mismo grupo que lidera nuestro sistema universitario e informa también sobre el estado del resto de las universidades. Por último la variedad de indicadores, períodos, campos y fuentes empleadas convierten a este producto 


\begin{tabular}{|c|c|c|c|c|c|c|c|c|c|c|c|c|}
\hline UNIVERSIDAD & MAT & FIS & QUI & $\mathrm{BIO}$ & MED & GEO & AGR & ING & TIC & PSI & ECO & OCS \\
\hline Alcalá de Henares & 32 & 28 & 30 & 29 & 21 & 11 & 23 & 40 & 27 & 15 & 37 & 29 \\
\hline Alicante & 15 & 16 & 10 & 40 & 28 & 16 & 29 & 17 & 26 & 31 & 13 & 22 \\
\hline Almería & 36 & -- & 17 & 37 & -- & 21 & 30 & 29 & 35 & 30 & 30 & 32 \\
\hline Autònoma de Barcelona & 6 & 3 & 6 & 4 & 2 & 2 & 1 & 2 & 21 & 5 & 2 & 3 \\
\hline Autónoma de Madrid & 7 & 4 & 5 & 2 & 3 & 13 & 21 & 10 & 13 & 3 & 9 & 14 \\
\hline Barcelona & 5 & 1 & 1 & 1 & 1 & 1 & 4 & 1 & 6 & 1 & 4 & 1 \\
\hline Burgos & -- & 35 & 28 & -- & -- & -- & -- & -- & -- & -- & -- & -- \\
\hline Cádiz & 38 & 37 & 33 & 36 & 34 & 28 & 12 & 28 & -- & 36 & -- & 41 \\
\hline Cantabria & 29 & 7 & -- & 35 & 18 & 36 & -- & 26 & 30 & -- & 33 & 25 \\
\hline Carlos III de Madrid & 12 & 19 & -- & -- & -- & -- & -- & 31 & 18 & -- & 3 & 2 \\
\hline Castilla La Mancha & 19 & 21 & 22 & 16 & 20 & 7 & 11 & 11 & 19 & 21 & 26 & 17 \\
\hline Complutense de Madrid & 14 & 5 & 4 & 6 & 5 & 4 & 2 & 3 & 14 & 6 & 20 & 4 \\
\hline Córdoba & -- & 39 & 23 & 20 & 12 & 23 & 6 & 24 & 20 & 37 & -- & - \\
\hline Coruña, A & 40 & 40 & 26 & 39 & 39 & 30 & 34 & 39 & 40 & 34 & 38 & 40 \\
\hline Deusto & -- & -- & -- & -- & -- & -- & -- & -- & -- & 20 & -- & -- \\
\hline Europea de Madrid & -- & -- & -- & -- & 40 & -- & -- & -- & -- & -- & -- & - \\
\hline Extremadura & 42 & 32 & 29 & 22 & 26 & 17 & 31 & 18 & 29 & 25 & 40 & 39 \\
\hline Girona & 35 & 24 & 18 & 28 & 36 & 14 & 26 & 21 & 24 & 41 & 31 & 20 \\
\hline Granada & 4 & 6 & 16 & 15 & 10 & 3 & 8 & 15 & 2 & 2 & 21 & 7 \\
\hline Huelva & -- & 30 & 35 & -- & -- & 24 & 36 & -- & -- & -- & -- & -- \\
\hline Islas Baleares & -- & 9 & 19 & 24 & 31 & 8 & -- & 36 & 32 & 19 & 28 & 9 \\
\hline Jaén & 34 & 31 & 34 & 34 & 37 & 37 & 38 & 35 & 25 & 18 & -- & - \\
\hline Jaume I de Castelló & 33 & 27 & 13 & -- & -- & 39 & -- & 33 & 22 & 11 & 25 & 16 \\
\hline La Laguna & 22 & 12 & 21 & 27 & 25 & 29 & 39 & 30 & 36 & 7 & 42 & 12 \\
\hline La Rioja & 41 & -- & 32 & -- & -- & -- & -- & -- & -- & -- & 41 & -- \\
\hline León & -- & -- & -- & 31 & 33 & 38 & 17 & -- & -- & 39 & -- & - \\
\hline Lleida & 37 & -- & 38 & 21 & 27 & 20 & 7 & 19 & 41 & 22 & -- & - \\
\hline Málaga & 16 & 29 & 25 & 30 & 22 & 22 & 27 & 23 & 12 & 17 & 35 & 37 \\
\hline Miguel Hernández & 26 & 38 & 39 & 19 & 14 & 35 & 13 & 34 & 38 & 27 & 34 & 38 \\
\hline Murcia & 25 & 23 & 20 & 17 & 19 & 27 & 5 & 27 & 31 & 10 & 17 & 26 \\
\hline Navarra & 31 & 36 & 36 & 12 & 6 & -- & 33 & 38 & 39 & 24 & 5 & 10 \\
\hline Oviedo & 18 & 18 & 12 & 10 & 11 & 25 & 24 & 20 & 11 & 12 & 22 & 18 \\
\hline Pablo Olavide & -- & -- & -- & 23 & 35 & 40 & -- & -- & -- & 38 & 19 & 34 \\
\hline País Vasco & 17 & 8 & 8 & 14 & 16 & 15 & 22 & 13 & 17 & 8 & 16 & 6 \\
\hline Palmas, Las & 39 & -- & -- & 41 & 23 & 31 & 28 & 42 & 37 & -- & 14 & 27 \\
\hline Politécnica de Cartagena & 28 & 41 & 41 & -- & -- & -- & 19 & 37 & 28 & -- & 29 & 42 \\
\hline Politècnica de Catalunya & 1 & 10 & 27 & 38 & 29 & 6 & 37 & 4 & 1 & 32 & 24 & 33 \\
\hline Politécnica de Madrid & 23 & 20 & 40 & 26 & 32 & 33 & 9 & 16 & 9 & 35 & 15 & 24 \\
\hline Politécnica de Valencia & 3 & 13 & 3 & 13 & 38 & 34 & 20 & 6 & 4 & 40 & 32 & 23 \\
\hline Pompeu Fabra & 11 & -- & - & 3 & 7 & -- & -- & -- & 8 & 13 & 1 & 5 \\
\hline Pública de Navarra & 30 & 33 & -- & 33 & -- & -- & 35 & 25 & 42 & 33 & 23 & 28 \\
\hline Ramon Llull & -- & -- & -- & -- & -- & -- & -- & -- & -- & -- & -- & 35 \\
\hline Rey Juan Carlos & -- & -- & -- & -- & 42 & -- & -- & -- & -- & -- & 39 & -- \\
\hline Rovira i Virgili & 20 & 22 & 11 & 25 & 15 & 10 & 14 & 14 & 16 & 29 & 27 & 13 \\
\hline Salamanca & 21 & 25 & 31 & 9 & 8 & 19 & 25 & 32 & 34 & 9 & 12 & 15 \\
\hline Santiago de Compostela & 2 & 14 & 7 & 7 & 13 & 12 & 3 & 12 & 15 & 16 & 10 & 21 \\
\hline Sevilla & 8 & 15 & 15 & 8 & 9 & 18 & 10 & 9 & 5 & 23 & 8 & 19 \\
\hline Uned & 27 & 34 & 37 & -- & 41 & -- & -- & 41 & 33 & 14 & 18 & 31 \\
\hline Valencia & 9 & 2 & 2 & 5 & 4 & 9 & 15 & 5 & 3 & 4 & 6 & 8 \\
\hline Valladolid & 24 & 26 & 24 & 32 & 24 & 32 & 32 & 22 & 23 & 28 & 36 & 30 \\
\hline Vigo & 13 & 17 & 14 & 18 & 30 & 5 & 16 & 8 & 7 & 26 & 7 & 36 \\
\hline Zaragoza & 10 & 11 & 9 & 11 & 17 & 26 & 18 & 7 & 10 & 42 & 11 & 11 \\
\hline
\end{tabular}

Tabla 3. Posiciones ocupadas por las universidades españolas en los 12 campos de conocimiento para el período 2005-2009 
en una herramienta para realizar comparaciones sólidas de interés para los gestores de la política universitaria.

\section{Notas}

\section{1. http://www.arwu.org/}

2. http://www.socialsciences.leiden.edu/cwts/productsservices/leiden-ranking-2010-cwts/

3. http://www.topuniversities.com/university-rankings/ world-university-rankings/home

4. http://www.timeshighereducation.co.uk/world-univer sity-rankings/

5. http://www.che-ranking.de/cms/?getObject=613\&get Lang=en

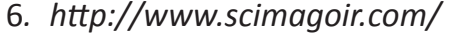

7. http://www.webometrics.info/

8. http://sci2s.ugr.es/rankinguniversidades/ http://rankinguniversidades.es/

9. Acrónimos de los campos científicos empleados en las tablas: MAT-Matemáticas, FIS-Física, QUI-Química, BIOCiencias biológicas, MED-Medica clínica, farmacia y farmacología, GEO-Ciencias geológicas y medioambientales, AGR-Ciencias agrarias, ING-Ingeniería, TIC-Tecnologías de la información y la comunicaciones, PSI-Psicología y educación, ECO-Economía, OCS-Otras ciencias sociales.

\section{Bibliografía citada}

Aguillo, Isidro F.; Bar-llan, Judit; Levene, Mark; Ortega, José-Luis. "Comparing university rankings". Scientometrics, 2010, v. 85 , n. 1, pp. 243-256.

Bordons, María; Sancho, Rosa; Morillo, Fernanda; Gómez, Isabel. "Perfil de actividad científica de las universidades españolas en cuatro áreas temáticas: un enfoque multifactorial". Revista española de documentación científica, 2010, v. 33, n. 1, pp. 9-33.

Buela-Casal, Gualberto; Bermúdez, María-Paz; Sierra, JuanCarlos; Quevedo-Blasco, Raúl; Castro, Ángel. "Ranking de
2009 en investigación de las universidades públicas españolas". Psicothema, 2010, v. 22, pp. 171-179.

Buesa, Mikel; Heijs, Joost; Kahwash, Omar. La calidad de las universidades en España. Elaboración de un índice multidimensional. Madrid: Consejo Económico y Social - Comunidad de Madrid, 2009.

Corera, Elena; Chinchilla, Zaida; De-Moya, Félix; SanzMenéndez, Luis. "Producción científica e impacto: ranking general y por áreas de las instituciones universitarias españolas". En: Informe CyD 2009. Barcelona: Fundación CyD, 2010, pp. 254-262.

Delgado-López-Cózar, Emilio; Jiménez-Contreras, Evaristo; Ruiz-Pérez, Rafael. "La ciencia española a través del Web of Science (1996-2007): las disciplinas". El profesional de la información, 2009a, v. 18, n. 4, pp. 437-443.

Delgado-López-Cózar, Emilio; Jiménez-Contreras, Evaristo; Ruiz-Pérez, Rafael. "España y los 25 grandes de la ciencia mundial en cifras (1992-2008)". El profesional de la Información, 2009b, v. 18, n. 1, pp. 81-86.

Liu, Nian Cai; Cheng, Ying. "Academic ranking of world universities - methodologies and problems ranking methodologies selection of universities". Higher education in Europe, 2005 , v. 30, n. 2, pp. 127-136.

Marginson, Simon; Van-der-Wende, Marijk. "To rank or to be ranked: the impact of global rankings in higher education". Journal of studies in international education, 2007, v. 11, n. 3-4, pp. 306-329.

Moed, Henk. Citation analysis in research evaluation. Dordrecht: Springer, 2005. ISBN 1402037139.

Ruiz-Pérez, Rafael; Delgado-López-Cózar, Emilio; JiménezContreras Evaristo. "Principios y criterios utilizados en España por la Comisión Nacional Evaluadora de la Actividad Investigadora (Cneai) para la valoración de las publicaciones científicas: 1989-2009". Psicothema, 2010. v. 22, n. 4, pp. 898-908.

Van Raan, Anthony F. J. "Fatal attraction: conceptual and methodological problems in the ranking of universities by bibliometric methods". Scientometrics, 2005, v. 62, n. 1, pp. 133-143.

El profesional de la información está indizada por la base de datos

ISI Social Science Citation Index, de Thomson Reuters, y según el

Journal Citation Report ha obtenido un Factor de impacto de 0,478 


\section{NOVEDADES EDITORIALES}

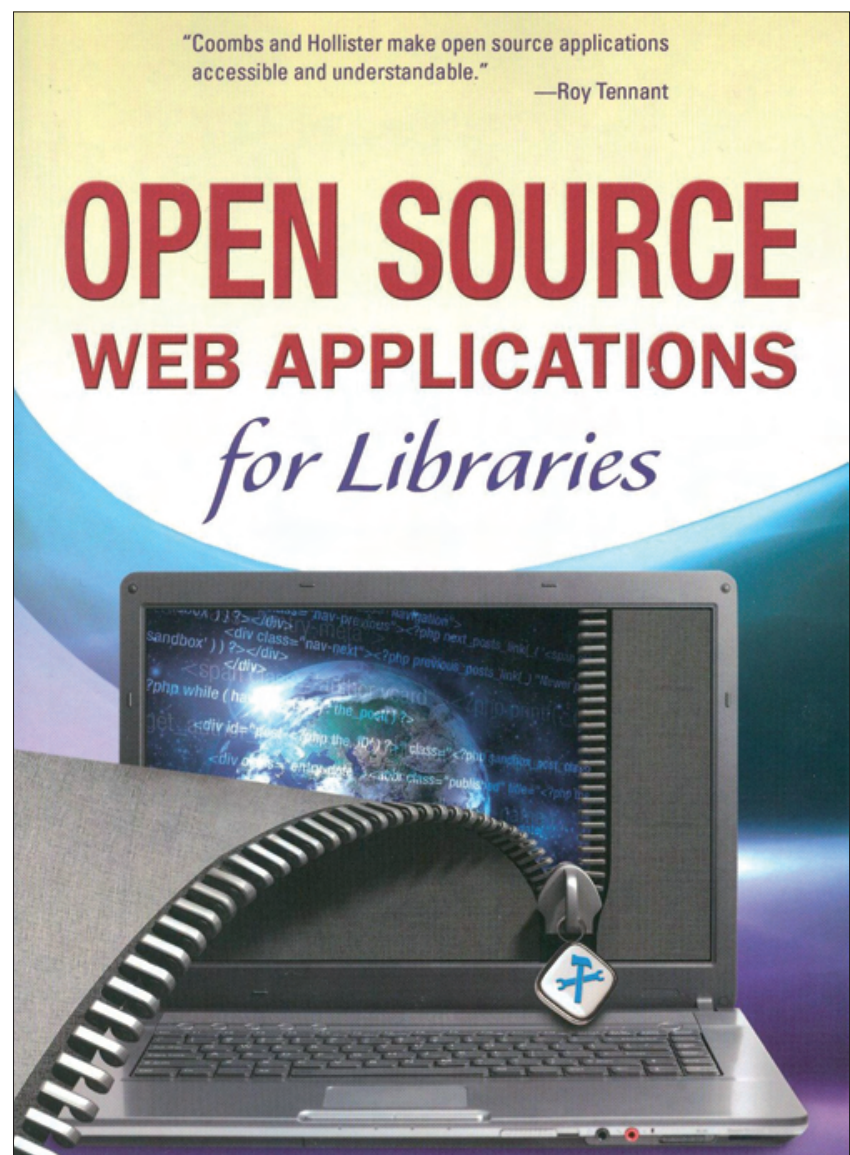

Karen A. Coombs \& Amanda J. Hollister

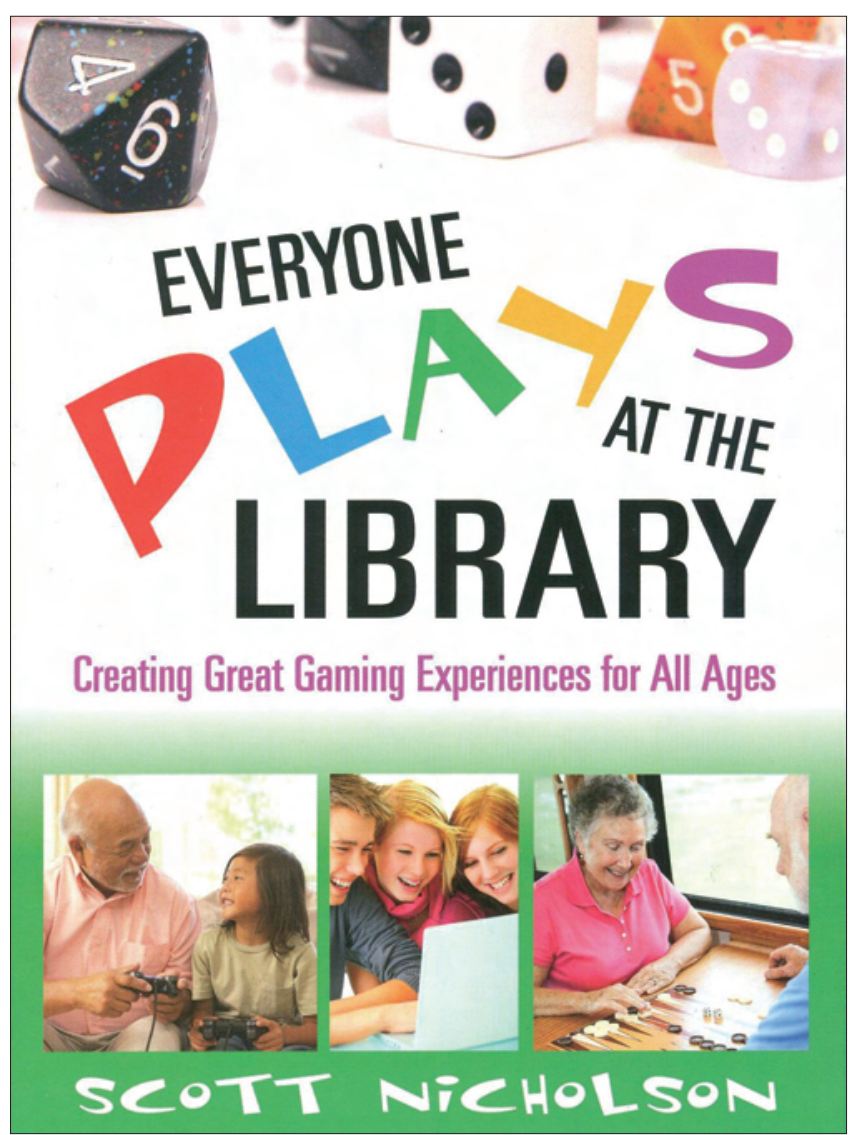

Open source web applications for libraries

Karen A. Coombs y Amanda J. Hollister

El interés por el software de código abierto nunca ha sido más fuerte, sin embargo hay falta de información sobre los programas que están disponibles, así como sobre sus beneficios -sin olvidar la preocupación sobre su fiabilidad y el soporte técnico- lo cual ha dificultado su adopción por parte de las bibliotecas. En este libro, Karen A. Coombs y Amanda J. Hollister abordan estas cuestiones y ofrecen a los bibliotecarios orientación sobre una serie de aplicaciones que pueden utilizarse para mejorar los servicios de referencia, alfin y divulgación de la biblioteca.

Además de explicar el uso, instalación y configuración de recursos tan populares como Wordpress, Drupal, Joomla y MediaWiki, los autores cubren aplicaciones de código abierto específicas para bibliotecas como SubjectsPlus, Blacklight, VuFind y Sopac. Las aplicaciones se comparan y contrastan, describiendo las principales características, fortalezas y debilidades con el fin de ayudar a los bibliotecarios a tomar decisiones informadas. Numerosos ejemplos reales muestran el uso de aplicaciones web de código abierto en diferentes tipos de bibliotecas.

2010, 280 pp, ISBN 978-1-57387-400-7. Precio: \$ 39,50

http://books.infotoday.com/

Everyone plays at the library. Creating great gaming experiences for all ages

Scott Nicholson

El libro Todos juegan en la biblioteca es ideal para aprender a organizar una sección de juegos. Scott Nicholson es educador, bibliotecario, diseñador de juegos, productor de la serie de vídeos "Juegos de mesa con Scott" y fundador del Laboratorio de Juegos de Biblioteca de Siracusa, muestra cómo implementar con éxito programas de juego en bibliotecas escolares, académicas y públicas, cubriendo todos los tipos de juegos para todas las edades.

El libro enseña a determinar las necesidades de los usuarios, alcanzar los objetivos de la biblioteca, obtener la aprobación de todos, llegar a los usuarios, crear una colección económica de grandes juegos, evaluar la efectividad del programa, juntar todas las edades, y crear el tipo de experiencia memorable de la que los usuarios hablen y deseen repetir.

2010, 248 pp. ISBN 978-1-57387-398-7. Precio: \$39,50 http://books.infotoday.com/ 

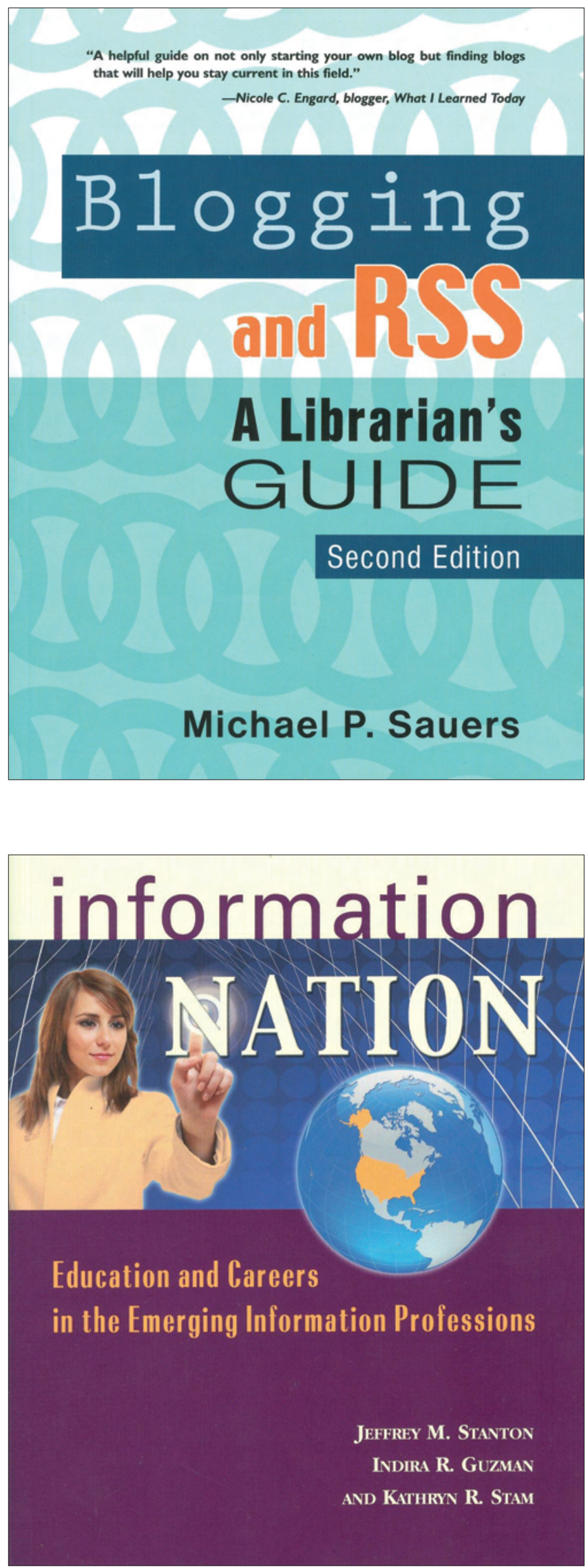

Blogging and RSS. A librarian's guide (2nd ed.) Michael P. Sauers

En esta segunda edición completamente actualizada de su popular libro de 2006, Michael P. Sauers, formador sobre temas de internet y blogger, muestra cómo las bibliotecas pueden utilizar fácilmente y con éxito la tecnología de los blogs y RSS. Sauers ofrece una gran cantidad de ejemplos e ideas útiles de bloggers bibliotecarios y proporciona instrucciones fáciles de seguir para la creación, publicación y sindicación de un blog a través de servicios gratuitos basados en la Web, software, canales RSS y agregadores. Esta segunda edición incluye nuevas herramientas y servicios para blogs, presenta numerosos blogs y bloggers bibliotecarios, e incluye un nuevo capítulo sobre micro-blogging con Twitter. Blogging and RSS es una lectura obligada para bibliotecarios, directores y administradores de biblioteca, personal técnico, y cualquier persona interesada en la utilización de blogs y RSS en un entorno de biblioteca.

2010, 336 pp. ISBN 978-1-57387-399-4. Precio: \$35,00 http://books.infotoday.com/

Information nation. Education and careers in the emerging information professions

Jeffrey M. Stanton, Indira R. Guzman y Kathryn R. Stam

Información y TICs son fundamentales para prácticamente todos los sectores, pero sin embargo las universidades no han logrado atraer, enseñar y producir una nueva generación de profesionales de la información que satisfaga la cada vez mayor necesidad de los mismos.

En este libro, tres educadores se preguntan por qué. Durante tres años analizaron a estudiantes y trabajadores de las profesiones de la información, poniendo de relieve los obstáculos a la inclusión y la retención de estudiantes de los EUA en los campos relacionados con la información. Se analizaron las fuerzas que impiden que los estudiantes de diplomatura y de grado obtengan las habilidades interdisciplinarias que necesitan para recuperar y conservar el liderazgo en el mundo de la información. Se narran los casos de diversos estudiantes que están prosperando en nuevos puestos de trabajo con potencial de revitalizar la economía.

2010, 225 pp., ISBN 978-1-57387-401-4. Precio: \$35,00 http://books.infotoday.com/ 


\section{EVALUADORES DE LA REVISTA 2009-2010}

Abadal-Falgueras, Ernest. Facultat de Biblioteconomia i Documentació, Universitat de Barcelona.

Aguillo, Isidro F. Centro de Ciencias Humanas y Sociales, Consejo Superior de Investigaciones Científicas, Madrid.

Aleixandre-Benavent, Rafael. Instituto de Historia de la Ciencia y Documentación López Piñero, Valencia.

Amat, Carlos B. Ingenio [CSIC-UPV], Institute of Innovation and Knowledge Management, Universidad Politécnica de Valencia.

Baiget, Tomàs. El profesional de la información, Barcelona.

Bustamante, Jesús. Centro Europeo para el Desarrollo de la Formación Profesional (Cedefop), Grecia.

Bustelo-Ruesta, Carlota. Inforárea SL, Madrid.

Caldera-Serrano, Jorge. Facultad de Biblioteconomía y Documentación, Universidad de Extremadura.

Calva-González, Juan-José. Centro Universitario de Investigaciones Bibliotecológicas, Universidad Nacional Autónoma de México.

Castillo-Vidal, Jesús. Baratz, Servicios de Teledocumentación SA, Madrid.

Codina, Lluís. Departamento de Comunicación, Universitat Pompeu Fabra, Barcelona.

D’Alòs-Moner, Adela. DOC6 Consultores en recursos de información, Barcelona.

Díaz-Noci, Javier. Departamento de Comunicación, Universitat Pompeu Fabra, Barcelona.

Del-Valle-Gastaminza, Félix. Facultad de Ciencias de la Información, Universidad Complutense de Madrid.

Delgado-López-Cózar, Emilio. Facultad de Comunicación y Documentación, Universidad de Granada.

Eíto-Brun, Ricardo. Grupo GMV y Universidad Carlos III de Madrid.

Fernández-Fernández-Cuesta, Paz. Instituto Juan March de Estudios e Investigaciones, Madrid.

Fernández-Molina, Juan-Carlos. Facultad de Comunicación y Documentación, Universidad de Granada.

Ferrer-Sapena, Antonia. Departamento de Comunicación Audiovisual, Documentación e Historia del Arte, Universidad Politécnica de Valencia.

Fidago, Antonio. Universidade da Beira Interior, Portugal.

Franganillo, Jorge. Facultat de Biblioteconomia i Documentació, Universitat de Barcelona.

Frías-Montoya, José Antonio. Departamento de Biblioteconomía y Documentación, Universidad de Salamanca.

Fuentes-Pujol, Eulàlia. Facultat de Ciències de la Comunicació, Universitat Autònoma de Barcelona.
Gallego-Linares, Elena. Gabinete Bibliográfico, Madrid.

García-Jiménez, Antonio. Facultad Ciencias de la Comunicación, Universidad Rey Juan Carlos, Madrid.

García-Marco, Francisco-Javier. Departamento de Ciencias de la Documentación, Universidad de Zaragoza.

García-Martín, Araceli. Agencia Española de Cooperación Internacional para el Desarrollo, Madrid.

Gómez-Hernández, José-Antonio. Facultad de Comunicación y Documentación, Universidad de Murcia.

Giménez-Toledo, Elea. Centro de Ciencias Humanas y Sociales, Consejo Superior de Investigaciones Científicas, Madrid.

González-Hernández, Cristina. Facultad de Ciencias de la Documentación, Universidad Complutense de Madrid.

Guallar, Javier. Facultat de Biblioteconomia i Documentació, Universitat de Barcelona; y Universitat Ramon Llull, Barcelona.

Hassan, Yusef. Grupo SCImago, Granada.

Keefer, Alice. Facultat de Biblioteconomia i Documentació, Universitat de Barcelona.

López-de-Quintana-Sáenz, Eugenio. Antena 3 TV, Madrid.

Marcos, Mari-Carmen. Departamento de Comunicación, Universitat Pompeu Fabra, Barcelona.

Marcos-Recio, Juan-Carlos. Facultad de Ciencias de la Información, Universidad Complutense de Madrid.

Martínez-San-Emeterio, Myriam. Grupo TecniPublicaciones, Madrid.

Masip-Masip, Pere. Facultat de Comunicació Blanquerna, Universitat Ramon Llull, Barcelona.

Méndez-Rodríguez, Eva. Departamento de Biblioteconomía y Documentación, Universidad Carlos III de Madrid.

McCathieNevile, Charles. Opera Software ASA, Noruega.

Mendo-Carmona, Concepción. Facultad de Ciencias de la Documentación, Universidad Complutense de Madrid.

Mendoza-García, Isabel. Biblioteca de la Escuela Técnica Superior de Ingenieros Industriales, Universidad Politécnica de Madrid.

Merlo-Vega, José-Antonio. Biblioteca, Universidad de Salamanca.

Micó-Sanz, Josep-Lluís. Facultat de Comunicació Blanquerna, Universitat Ramon Llull, Barcelona.

Michel, Pepa. Biblioteca Nacional de España, Madrid.

Millán, José-Antonio. Libros y bitios, JAMillán.com, Barcelona.

Moreiro-González, José-Antonio. Departamento de Biblioteconomía y Documentación, Universidad Carlos III de Madrid. 
Muñoz-de-Solano-Palacios, Bárbara. Ministerio de Defensa, Madrid.

Muñoz-Tinoco, Concha. Biblioteca, Hospital Ramón y Cajal, Madrid.

Noguera, José-Manuel. Universidad Católica San Antonio, Alicante.

Orduña-Malea, Enrique. Escuela Técnica Superior de Informática, Universidad Politécnica de Valencia.

Palacios, Marcos. Universidade Federal da Bahia, Brasil.

Pascual-Miguel, Félix-José. E.T.S. Ingenieros de Telecomunicación, Universidad Politécnica de Madrid.

Pérez-Montoro, Mario. Facultat de Biblioteconomia i Documentació, Universitat de Barcelona.

Pedraza-Jiménez, Rafael. Departamento de Comunicación, Universitat Pompeu Fabra, Barcelona.

Peset, Fernanda. Departamento de Comunicación Audiovisual, Documentación e Historia del Arte, Universidad Politécnica de Valencia.

Ramírez-Martín, Susana M. Facultad de Ciencias de la Documentación, Universidad Complutense de Madrid.

Reyes-Pacios, Ana. Departamento de Biblioteconomía y Documentación, Universidad Carlos III de Madrid.

Ribeiro, Fernanda. Departamento de Jornalismo e Ciências da Comunicação, Faculdade de Letras, Universidade do Porto, Portugal.

Rodríguez-Gairín, Josep-Manuel. Facultat de Biblioteconomia i Documentació, Universitat de Barcelona.

Rodríguez-Yunta, Luis. Centro de Ciencias Humanas y Sociales, Consejo Superior de Investigaciones Científicas, Madrid.
Rovira, Cristòfol. Departamento de Comunicación, Universitat Pompeu Fabra, Barcelona.

Salaverría, Ramón. Facultad de Comunicación, Universidad de Navarra.

Sancho, Rosa. Centro de Ciencias Humanas y Sociales, Consejo Superior de Investigaciones Científicas, Madrid.

Sanz-Casado, Elías. Departamento de Biblioteconomía y Documentación, Universidad Carlos III de Madrid.

Sánchez-Jiménez, Rodrigo. Facultad de Ciencias de la Documentación, Universidad Complutense de Madrid.

Sánchez-Vigil, Juan-Miguel. Facultad de Ciencias de la Documentación, Universidad Complutense de Madrid.

Seal, Robert. Loyola University, Chicago, EUA.

Spinak, Ernesto L. Consultor, Montevideo, Uruguay.

Tejada-Artigas, Carlos-Miguel. Facultad de Ciencias de la Documentación, Universidad Complutense de Madrid.

Torres-Salinas, Daniel. Centro de Investigación Médica Aplicada, Universidad de Navarra; y EC3, Universidad de Granada.

Torres-Padrosa, Víctor. Universitat Politècnica de Catalunya, Barcelona.

Tosete-Herranz, Francisco. Consultor en sistemas de información, bibliotecas digitales y usabilidad, Madrid.

Tramullas, Jesús. Departamento de Ciencias de la Documentación, Universidad de Zaragoza.

Vergueiro, Waldomiro. Escola de Comunicações e Artes, Universidade de São Paulo, Brasil.

\section{EPI $n^{\circ} 1$ en el ranking In-Recs $Y$ el Anuario ThinkEPI se estrena con la $7^{\mathrm{a}}$ posición}

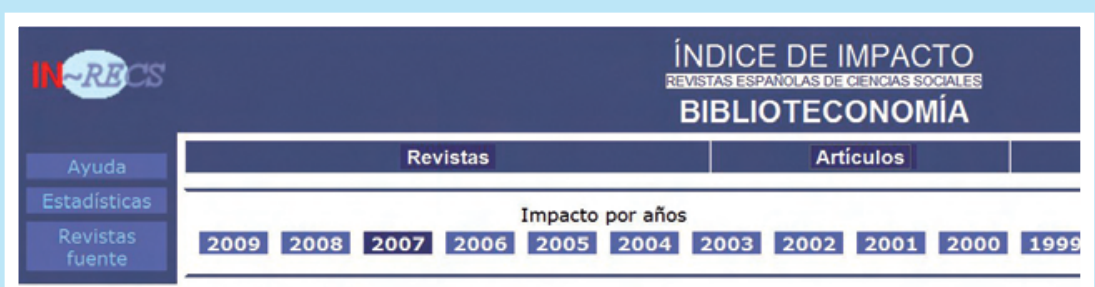

ÍNDICE DE IMPACTO: 2009 Población de revistas: 26

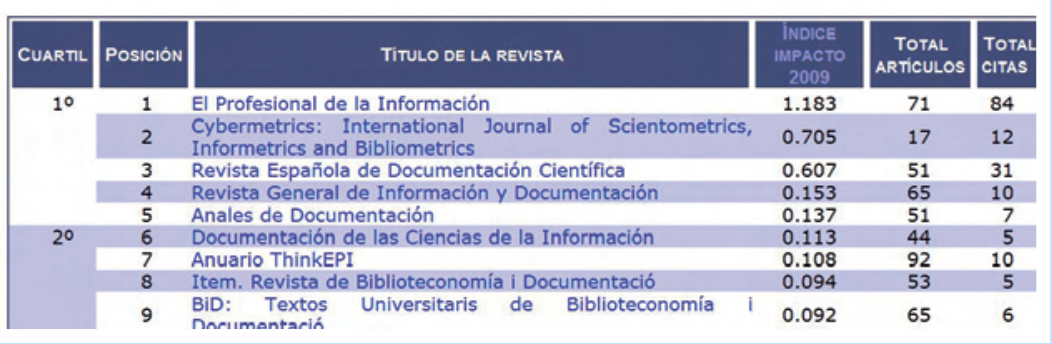

Según el Índice de Revistas Españolas de Ciencias Sociales (In-Recs), elaborado por el Grupo EC3 (Evaluación de la Ciencia y de la Comunicación Científica) de la Universidad de Granada: http://ec3.ugr.es/in-recs/ii/ Biblioteconomia-fecha-2009. htm la revista El profesional de la información ha conseguido situarse en primer lugar, de entre 26 revistas españolas de biblioteconomía y documentación. 